\title{
LA VINCULACIÓN EN LAS UNIVERSIDADES PÚBLICAS VERSUS LAS UNIVERSIDADES PRIVADAS EN MÉXICO. RETOS Y DESAFÍOS EN EL SIGLO XXI ${ }^{1}$
}

\author{
Adria Velia González Beltrones, ${ }^{2}$ Guadalupe Aleida Valenzuela Miranda, ${ }^{3}$ \\ María Dolores Rocha Ontiveros, ${ }^{4}$ Kevin Ricardo Ortíz, Erika Anahiza Cázares ${ }^{5}$
}

\section{Resumen}

En el presente documento se identifican algunas de las formas de vinculación-entorno social de las Universidades Públicas, su comparación con la vinculación de las Universidades Privadas y sus posibles soluciones potenciales desde la academia .

Para la obtención de información se tomaron los datos de varios instrumentos aplicados siguiendo a Casalet y Casas (1996), con el objetivo de explorar las características y vinculación entre la Universidad y el sector público, privado y social desde el punto de vista de las Instituciones de Educación Superior.El desafío de las Universidades Públicas y o Privadas es confirmar su compromiso con la sociedad de modelar los recursos humanos que la misma necesita, para ello es imprescindible estrechar la brecha entre lo que se enseña en las Universidades y lo que demanda los sectores laborales público y privado y social, por lo que a partir del monitoreo de la evolución de dichos sectores se deberá realizar los ajustes necesarios al currículo de los diferentes programas de estudios como una respuesta a esas necesidades. Lo que facilitará la vinculación vía proyectos institucionales con el sector público, privado y social.

Palabras claves: vinculación, desafíos institucionales, sectores público, privado y social

\section{Introducción}

De acuerdo a los expertos, la vinculación entre empresas e instituciones de educación superior (IES) podría considerarse como una relación natural, dada la

\footnotetext{
1 Ponencia presentada en el VII Congreso internacional RIAICES 2017 Impacto de la investigación en docencia e innovación en la calidad de la formación en Educación Superior. Chillán,Chile 2 al 4 de noviembre de 2017, Universidad del Bío-Bío.

${ }^{2}$ Docente Investigadora, Departamento de Derecho,DCS,URC, Universidad de Sonora, México

3 docente Investigadora, Departamento de Derecho,DCS,URC, Universidad de Sonora, México

${ }^{4}$ Docente Investigadora, D de Sociología y Administración Pública, DCS,URC, Universidad de Sonora, México

${ }^{5}$ Estudiantes de la Licenciatura en Derecho y Ayudantes en el Área de Investigación de los CAEC del DDDCS URC Universidad de Sonora
} 
complementariedad que se puede esperar entre estos dos sectores de la economía (Porter 1979; 1980) en términos del potencial de las IES para proveer los recursos humanos adecuados, las soluciones tecnológicas desarrolladas en las aulas y/o laboratorios y áreas de investigación, requeridos a su vez por los sectores productivos, públicos y privados así como por el amplio sector social de un país. Sin embargo, la vinculación --por lo menos en México-- entre las empresas y las instituciones de educación superior, no se ha dado en forma natural sino de manera inercial, es decir, en tanto que para las empresas, la vinculación con las IES podría significar una mas de las múltiples opciones o medios para lograr una mayor efectividad en el incremento de la productividad, posicionamiento en el mercado, crecimiento e incremento de las utilidades, entre otros. En contrapartida, para las IES Públicas en Latinoamérica la vinculación comenzó a concretar su campo de acción en lo social y lo cultural y de acuerdo a las características socioculturales y económicas de cada región, así como de sus circunstancias históricas específicas, permaneciendo durante mucho tiempo en actitud pasiva respecto a la vinculación en el sentido de preparar inercialmente recursos humanos en las actividades tradicionalmente necesarias para la vida en la comunidad (vbg. Ingenieros, abogados, arquitectos, médicos, contadores públicos entre otras)manejándose conservadoramente respecto a los cambios tecnológicos y socioeconómicos en el diseño de nuevas licenciaturas al efecto. Por su parte, las IES privadas de manera mas natural desarrollaron la vinculación con el sector empresarial dado que en la práctica generaban los recursos humanos visiblemente encaminados a satisfacer las demandas de este. Es en este sentido, que en el sector privado, involucrado en contender con la competencia de empresas similares, la vinculación derivaría en una estrategia inducida por la estructura de los mercados. Y por ello el pretender vincularse sería para contratar personal con perfil determinado, para adquirir tecnología, o bien para desarrollar o financiar investigación específica. Es en este sentido que las empresas se vinculan con las IES en la medida en que el contexto se los demande, como un mecanismo para alcanzar sus propios fines y viceversa en cuanto a las IES y sus fines a alcanzar se refiere. 


\section{Objetivo}

identificar algunas de las formas de vinculación-entorno social de las Universidades Públicas, su comparación con la vinculación de las Universidades Privadas y sus posibles soluciones potenciales desde la academia .

\section{Justificación}

Señalan los expertos que el término extensión o vinculación universitaria tendrá una connotación diferenciada acorde a las distintas formas de interacción de las universidades con la sociedad, sus organizaciones e instituciones, o en su caso, las distintas percepciones de la universidad en si misma, del conocimiento y del contexto en el que se desenvuelven.

\section{A manera de Hipótesis}

Para la universidad pública latinoamericana: la función social, significa " poner el saber universitario al servicio de la sociedad y hacer de sus problemas un aspecto importante de su ocupación"(González y González, 2013, p. 6). Por su parte, las IES privadas de manera natural desarrollan la vinculación con el sector empresarial

\section{Marco Teórico}

\section{Formación de la Función Social de la Universidad en América Latina}

La discusión en relación al "deber ser" de esta vinculación entre Universidad/Sociedad, ha sido a nivel de la reflexión, del discurso y de la práctica:

\footnotetext{
${ }^{6}$ En esencia, el planteamiento básico parte del supuesto económico originado en la premisa de que toda "universidad pública, en última instancia es pagada por el esfuerzo de la comunidad, y por ello, aportará a la sociedad por medio de la extensión universitaria, llevada a cabo fundamentalmente por los estudiantes " (González y González, 2013, p. 6).
} 
"la función social de la Universidad ha sido abordada de acuerdo a las características sociales, culturales y económicas de cada región, así como de sus circunstancias históricas especificas." (URL, 2004, p. 5).

En América Latina, el tema fue enunciado por primera vez bajo el término de "extensión universitaria", en el Primer Congreso Internacional de Estudiantes Americanos en Montevideo (1908), donde quedaron las bases de lo que posteriormente fuera :

- la Reforma de Córdoba (1918), una importante propuesta elaborada por estudiantes, con la cual se fortaleció el postulado de la función social de la Universidad Latinoamericana y se generaron una gama de actividades que definieron el perfil de la Universidad Latinoamericana, cuyo impacto llegó a Centroamérica en 1931 y prevalece hasta nuestros días. (URL, 2004, p. 6).

Posteriormente, en 1949 se realizó el Primer Congreso de Universidades Latinoamericanas en Guatemala, en donde se aprobaron una serie de resoluciones relacionadas con el concepto mismo de la Universidad en América Latina.

En cuanto a la extensión universitaria, se marcó la intención de proyectar, el quehacer universitario a todas las esferas sociales que constituyen la realidad nacional.

La extensión debía abarcar el campo de los conocimientos científicos, literarios y artísticos, utilizando todos los recursos que la técnica contemporánea permitía poner al servicio de la cultura.

Así pues, en algunas universidades, la extensión universitaria empieza a concretar su campo de acción en dos ramas: lo social y lo cultural.

Posteriormente, en 1957 se convocó a la Primera Conferencia Latinoamericana de Extensión Universitaria y Difusión Cultural, en Chile, en donde se aprobaron una serie de recomendaciones destinadas a precisar la teoría latinoamericana sobre esta materia.

La definición de extensión universitaria en ese momento fue: (URL, 2004, p. 6).

- "La extensión debe ser conceptuada por su naturaleza, contenido, procedimientos y finalidades..." (Super, s.f. citado en URL, 204, p. 6). 


\section{Vinculación de las Universidades Europeas}

Las universidades europeas en sus orígenes se caracterizaron por dedicarse exclusivamente a la formación de una parte de los integrantes de las capas dominantes como una vía de mantener "el poder del saber", limitado a una pequeña porción de los miembros de la sociedad.

No obstante, a mediados del Siglo XIX la universidad medieval europea, centrada en el proceso de enseñanza, tomó su papel como institución generadora de conocimientos a través del principio de unidad entre docencia e investigación, en lo que se denomina por algunos autores como la "primera revolución académica".

Tal situación, amplió el vínculo con el entorno y los principios de autonomía y financiamiento público de las actividades científicas, atravesaron el desarrollo universitario y se afianzaron durante más de 50 años.

Las universidades europeas, dedicadas al proceso docente y de investigación, desarrollaban de manera muy esporádica algunas actividades relacionadas fundamentalmente con las bellas artes. (González y González, 2013, p.

En el mismo sentido, cabe destacar que la necesidad de adaptarse a la dinámica económica y competitiva de diversos contextos nacionales, a fines del pasado siglo, puso de manifiesto que la investigación científica básica no era suficiente para promover el desarrollo tecnológico, lo que provocó la búsqueda de nuevas vías al emprender los procesos de generación y difusión de conocimientos.

En este periodo emergen nuevos enfoques que llevan implícitos cambios importantes en la organización y desempeño de las universidades, en particular en cuanto a la estructura organizativa y sus vínculos con el entorno (Castro y Vega, 2009, p. 7273).

Asimismo, se subraya que tales desarrollos llevaron a la valoración de que las universidades europeas se encontraban ante una "segunda revolución académica", la que ha desembocado en la adopción de lo que han dado en llamar " 'tercera misión', que abarca todas aquellas actividades relacionadas con la generación, uso, aplicación y explotación, fuera del ámbito académico, del conocimiento y de otras 
capacidades de las que disponen las universidades " (Molas-Gallart, Salter, Patel, Scott y Durán, 2002).

El cumplimiento de esta "tercera misión" ha llevado a la universidad a convertirse en un actor determinante en los procesos de desarrollo social y económico, a través de un vínculo más estrecho con su entorno.

En este nuevo modelo de universidad, actividades como la transferencia de conocimiento o la incubación de empresas, dejan de ser casuales y se convierten en permanentes, y van impregnando los estamentos universitarios.

Esto genera un círculo virtuoso, donde las empresas se ven favorecidas por una mayor competitividad y las universidades perciben los beneficios de integrarse en la sociedad mediante un nuevo contrato social, el cual, a diferencia del anterior, demanda un mayor direccionamiento de las actividades de investigación hacia las necesidades sociales.

En contrapartida a la visión eurocentrista, otros autores consideran que las causas que llevan a las universidades latinoamericanas a cambiar sus políticas de ciencia y tecnología, no tienen su origen en la experiencia de los países desarrollados, sino en la realidad neoliberal, que reduce a niveles mínimos el financiamiento de las universidades y las compulsa a la búsqueda de recursos por otras vías [...]. (González y González, 2013, p. 7).

Tampoco se identifican con el planteamiento de que esta situación "promueve la adopción de la tercera misión", ya que esto contradice lo expresado por los propios autores acerca de la marcada vocación extensionista de las universidades latinoamericanas, las cuales, desde principios del siglo XX, tienen su "tercera misión", por tanto no necesitan adoptar lo que ya tienen. Respecto al planteamiento acerca de las reticencias internas provocadas por estos cambios, se valora que efectivamente la adopción del modelo economicista obliga a la universidad a asumir prácticas no 
tradicionales y a abordar nuevos aspectos que pueden alejarla de la tradición y someterla a los dictados del mercado, pero se ha asumido como "mal necesario" dada la perentoria necesidad de recursos económicos. (González y González, 2013, p. 7).

\section{Metodología}

Para la obtención de información del caso que nos ocupa, siguiendo a Casalet y Casas $1996^{7}$, se tomaron los datos de varios instrumentos aplicados, con el objetivo de explorar las características de la vinculación entre las Universidades Públicas y Privadas y el sector público, privado y social desde el punto de vista de las Instituciones de Educación Superior IES en México.

\section{Comparativo de las Características de la vinculación entre las IES Públicas y Privadas y el sector público, privado y social}

1) Universidad de Guadalajara/ Consorcio de Investigación sobre México PROFMEX con UCLA en 2014, realizaron una encuesta sobre vinculación de Investigadores en Ciencia y Tecnología de México, para identificar la situación que guarda la vinculación de investigadores en ciencia y tecnología con empresas, gobiernos y organizaciones no gubernamentales, así como su potencial para el desarrollo socioeconómico desde su propio punto de vista.

Su Hallazgo: Alto porcentaje de las instituciones declararon realizar actividades de vinculación tanto en lo formal como en lo informal.

2) El Centro de Investigación y Docencia Económicas CIDE por encargo de la

\footnotetext{
${ }^{7}$ Realizaron una encuesta sobre vinculación en 1996 para conocer la colaboración entre academia y empresa. Su informe analiza los datos proporcionados por 247 instituciones; 78 de ellas organismos universitarios públicos, 42 particulares; 63 institutos tecnológicos, 33 instituciones de investigación públicas y 27 institutos privados. Su hallazgo :alto porcentaje de las instituciones realizan actividades de vinculación de manera formal e informal.
} 
Secretaría de Educación Pública SEP (2010a) realizó la Encuesta Nacional de Vinculación ENAVI a encargados de oficinas de vinculación sobre situación y percepción en 351 Instituciones de Educación Superior IES del país y la Encuesta Nacional de Vinculación con Empresas ENAVES (2010b) sobre condiciones y percepciones de las empresas en 351 Instituciones de Educación Superior IES del país. Sus hallazgos:

a)Las IES(públicas y privadas) y las empresas en su mayoría, realizan vinculación mediante actividades llevadas a cabo con el fin de desarrollar proyectos relacionados con la formación y transferencia de recursos humanos. Estas actividades, ${ }^{8}$ son consideradas de "baja complejidad" y forman una de las modalidades de cooperación reportada con mayor frecuencia en la encuesta a nivel nacional.

b) Igualmente se establece la vinculación para la transferencia de recursos técnicos entre universidades y empresas, mediante actividades que implican una mayor complejidad al obtener servicios de asistencia y asesoría técnica y administrativa, en temas como la gestión de la calidad, la obtención de certificaciones o el logro de estándares industriales, la regulación comercial, la formulación de proyectos y el desarrollo de planes de negocio, entre otros.

La mitad (54.54\%) de las IES realiza actividades de investigación, desarrollo experimental o innovación no comisionados por empresas u organismos.

El $36.17 \%$ de las IES encuestadas ofrecen servicios tecnológicos al mismo sector.

Sólo 16.31\% contaría con incubadoras de empresas.

\footnotetext{
8 tales como: implementación de una bolsa de trabajo, realización de pasantías de estudiantes en las empresas participantes, realización de prácticas profesionales de estudiantes en la empresa, concertación de estadías/residencias profesionales de académicos en la empresa, realización de proyectos de servicio social, capacitación técnica o administrativa, iniciación de proyectos de educación continua, formación de postgrados, realización de intercambio de personal entre otras.
} 
BIOLEX Revista Jurídica del Departamento de Derecho UNISON URC

Academia de Derecho Administrativo

Tercera Época Año 9. No 17 Julio -Diciembre de 2017

El $68.09 \%$ de las IES encuestadas reportan haber prestado servicios de consultoría.

c) La Vinculación para la transferencia de recursos para investigación $y$ desarrollo se observa mediante actividades de colaboración más compleja ${ }^{9}$ entre la industria y las IES, para transferir recursos destinados al desarrollo de investigación y gestión. El $68.09 \%$ de las IES encuestadas reportan haber prestado servicios de consultoría. Las actividades de vinculación se realizan con mayor frecuencia con universidades e institutos tecnológicos y en menor medida con centros de investigación.

Cabe destacar la mención de la reducción significativa de instituciones y empresas que establecen vínculos para desarrollar proyectos de vinculación en esta tercera categoría, que corresponde a la de mayor complejidad entre las modalidades precitadas .

Asimismo la ENAVI confirma la existencia de un mercado de consultores que desplazaría a las IES como interlocutores en el establecimiento de vínculos con las empresas para el desarrollo de certificaciones y actividades destinadas a impactar en procesos productivos. De igual manera, este último punto confirmaría otros análisis realizados (CIDE/SEP, 2010a) en los que se reportó una relación negativa entre el grado de complejidad de las actividades llevadas a cabo y la frecuencia con la que las instituciones educativas reportaban participar en su organización y realización.

\footnotetext{
${ }^{9}$ transferencia de recursos para actividades de investigación y gestión en general, desarrollo de investigación conjunta, asistencia a foros académicos, participación en foros empresariales, foros público-privados, o bien participación de empresas en cuerpos directivos de instituciones de educación superior, conforme a las categorías consideradas en el cuestionario de la ENAVI.
} 
3) ONU (2015) Encuesta "My World", vía internet, telefonía móvil e impresa hizo llegar las Naciones Unidas, a los líderes mundiales y al Grupo de Alto Nivel del Secretario General, opinión de miles de personas sobre los problemas fundamentales que debería abordar la futura agenda global del desarrollo ${ }^{10}$.

\section{Resultados y discusión}

Siguiendo a González y González, 2013, p. 7) La adopción del modelo economicista obliga a la universidad pública a asumir prácticas no tradicionales y a abordar nuevos aspectos que pueden alejarla de la tradición y sujetarse a los dictados del mercado, en contrapartida para las universidades privadas tal modelo les resulta bastante cómodo, lo que en el caso que nos ocupa se ha verificado según las deducciones de los estudios base del presente.

Aun cuando el común denominador en las IES del sector público como del privado lo es la necesidad de recursos económicos, ciertamente ambas modalidades requieren sumarse al esfuerzo de los gobiernos nacional y supranacional por alcanzar los Objetivos del Milenio extendidos ${ }^{11}$ a la Nueva Agenda de Desarrollo Sostenible 2030

\footnotetext{
${ }^{10}$ Más de 8 millones de personas ---agosto de 2015--- de más de 190 países habían "votado" por sus principales prioridades para el desarrollo para contribuir con eficacia y eficiencia al desarrollo sustentable de la sociedad, y sumarse al logro de los Objetivos del Milenio al 2015, actualmente extendidos al 2030, en pro de cuya obtención, el Secretario General de la ONU, se encuentra en campaña permanente

${ }^{11}$ Poner fin a la pobreza en todas sus formas en todo el mundo. Poner fin al hambre, lograr la seguridad alimentaria y la mejora de la nutrición y promover la agricultura sostenible. Garantizar una vida sana y promover el bienestar para todas y todos en todas las edades. Garantizar una educación inclusiva, equitativa y de calidad y promover oportunidades de aprendizaje durante toda la vida para todas y todos. Lograr la igualdad entre los géneros y empoderar a todas las mujeres y niñas. Garantizar la disponibilidad de agua y su gestión sostenible y el saneamiento para todos. Garantizar el acceso a una energía asequible, segura, sostenible y moderna para todos. Promover el crecimiento económico sostenido, inclusivo y sostenible, el empleo pleno y productivo y el trabajo decente para todos. Construir infraestructura resiliente, promover la
} 
asumiendo un papel más activo en la consecución de los posibles planes de acción, adecuando al efecto, los contenidos de los programas de sus planes de estudio en las diversas disciplinas, formando recursos humanos capaces de integrarse en equipos disciplinares o interdisciplinares que busquen o gestionen las mejores soluciones a los múltiples problemas sociales, económicos y ambientales que existen en nuestro entorno local, regional, nacional y global, sin soslayar el promover en las aulas y en los espacios de investigación, la innovación tecnológica, el desarrollo de las competencias de innovación básicas, de creación de empresas o productos de alto nivel tecnológico, de gestión y registro de patentes, mecanismos de protección de marcas y derechos de autor, desarrollo de incubadoras o aceleradoras de negocios y de ecosistemas de innovación regional, entre otras tareas con las que la comunidad universitaria puede contribuir, a concienciar sobre los problemas sociales que existen en nuestro planeta y a su solventación.

Por todo lo anterior, cabe mencionar que el tema de la "extensión universitaria," dentro de las IES Públicas y Privadas en nuestro país, "debiera atenderse con visión integradora, con la misma urgencia con la que se requiere intentar erradicar la brecha económica ominosa entre los que tienen recursos económicos en exceso y los que carecen de ellos, si bien tomando en cuenta las externalidades que condicionan su enfoque, acorde también al compromiso asumido del desarrollo sostenible para todos, y

industrialización inclusiva y sostenible y fomentar la innovación. Reducir la desigualdad en y entre los países. Lograr que las ciudades y los asentamientos humanos sean inclusivos, seguros, resilientes y sostenibles. Garantizar modalidades de consumo y producción sostenibles. Adoptar medidas urgentes para combatir el cambio climático y sus efectos. Conservar y utilizar en los océanos, los mares y los recursos marinos para el desarrollo sostenible. Promover el uso sostenible de los ecosistemas terrestres, luchar contra la desertificación, detener e invertir la degradación de las tierras y frenar la pérdida de la diversidad biológica. Promover sociedades pacíficas e inclusivas para el desarrollo sostenible, facilitar el acceso a la justicia para todos y crear instituciones eficaces, responsables e inclusivas a todos los niveles. Fortalecer los medios de ejecución y revitalizar la Alianza Mundial para el Desarrollo Sostenible. 
en la medida de sus posibilidades tecnológicas y de sus recursos económicos y humanos." (González,B.,Valenzuela, M., y Rocha O. 2016,p141)

El desarrollo histórico del término extensión, plasmado en este documento tanto de las universidades europeas, las universidades latinas y centro americanas permite plantear la necesidad de una transición de su concepto, desde una visión estrecha a una conceptualización más amplia, acorde a las distintas formas de interacción de las universidades con la sociedad, sus organizaciones e instituciones,

\section{A manera de conclusiones}

De los contenidos de las estudios base del presente documento, se confirman las hipótesis planteadas en relación a :

- La evolución de la vinculación de las IES Públicas en México desde la marcada vocación extensionista de las universidades latinoamericanas, de principios del siglo XX, a la adopción forzada del modelo economicista y con ello convertirse en un actor determinante en los procesos de desarrollo social y económico, a través de un vínculo más estrecho con su entorno, mediante un nuevo contrato social, que le demanda un mayor direccionamiento de las actividades de investigación hacia las necesidades sociales y

- Asimismo se confirma la hipótesis en relación a la manera natural en que las IES privadas desarrollan la vinculación con el sector empresarial

De lo anterior se desprende que el desafío de las Universidades Públicas y Privadas es:

- Confirmar su compromiso con la sociedad, de modelar los recursos humanos que la misma necesita,

Para ello es imprescindible:

- Estrechar la brecha entre lo que se enseña en las Universidades Públicas y Privadas y lo que demanda..... los sectores laborales público privado y social, 
BIOLEX Revista Jurídica del Departamento de Derecho UNISON URC

Academia de Derecho Administrativo

Tercera Época Año 9. No 17 Julio -Diciembre de 2017

El desafío de las IES y Privadas, a partir del monitoreo de la evolución de los sectores público, privado y social y de lo imperativo de ser agentes activos para la consecución de los Objetivos del Milenio extendidos a la Nueva Agenda de Desarrollo Sostenible 2030 es:

- Realizar los ajustes necesarios al currículo de los diferentes programas de estudios, en respuesta a esas necesidades.

- Lo que facilitará la vinculación vía proyectos institucionales con el sector público, privado y social.

\section{Bibliografía}

Bárcena, A. (2016). Prólogo. En CEPAL, Horizontes 2030: la igualdad en el centro del desarrollo sostenible. Ciudad de México: CEPAL. Recuperado de:

http://www.infolatam.com/2016/05/22/ horizontes-2030-la-igualdad-en-el-centro-del-desarrollosostenible/

Casalet, M., y Casas, R. (1998). Un diagnóstico sobre la vinculación Universidad-Empresa Conacyt-Anuies. Ciudad de México: Asociación Nacional de Universidades e Instituciones de Educación Superior y Consejo Nacional de Ciencia y Tecnología,Reseña. Recuperado de: http://publicaciones.anuies. mx/revista/107/3/1/es/un-diagnostico-sobre-la-vinculacionuniversidad-empresa-conacyt

Castro, E., y Vega, J. (2009). Las relaciones universidad-entorno socioeconómico en el Espacio Iberoamericano del Conocimiento, Revista CTS, 4(12), 71-81. Recuperado de: http://www. scielo.org.ar/pdf/cts/v4n12/v4n12a08.pdf

Del Huerto, M.E. (2007). La Extensión Universitaria como vía para fortalecer los vínculos Universidad- Sociedad desde la promoción de salud. Revista cubana de Salud Pública, 33(2). Recuperado de: http://scielo.sld.cu/scielo.php?script=sci_arttext\&pid=S086434662007000200005

González, M., y González, G. R. (2013). ¿Extensión universitaria,proyección social o tercera misión?una reflexión necesaria. Revista Congreso Universidad, II(2). Recuperado de: http://www. congresouniversidad.cu/revista/index.php/congresouniversidad/article/view/151

González B.,Valenzuela M., y Rocha O. (2016) Barreras institucionales que obstaculizan al interior de las IES el desarrollo académico y su vinculación con la sociedad en Valenzuela Blanca Aurelia, et.al ,(2016) Educación y Universidad ante el Horizonte 2020, Inclusión y cultura colaborativa entre empresa y sociedad Volumen 1, México, lera. Edición, Ed. Qarttuppi 
BIOLEX Revista Jurídica del Departamento de Derecho UNISON URC

Academia de Derecho Administrativo

Tercera Época Año 9. No 17 Julio -Diciembre de 2017

Landivar Rafael (2004). Políticas Institucionales: Responsabilidad Social Universitaria.

Recuperado de: http://www.url.edu.gt/portalurl/archivos/89/archivos/pol_inst_rsu.pdf

Molas-Gallart, J., Salter, A., Patel, P., Scott, A., \& Duran, X. (2002). Measuring third stream activities. England: SPRU. University of Sussex. Recuperado de:

https://www.academia.edu/532097/ Measuring_third_stream_activities

Organización de las Naciones Unidas (ONU). (2015). Objetivos de Desarrollo sostenible.

Recuperado de: http://www.onu.org.mx/agenda-2030/objetivos-del-desarrollo-sostenible/

Porter, Michael (1980), Competitive Strategy: Techniques for analyzing industries and competitors, Nueva York/Londres, Free Press.

Secretaría de Educación Pública/ Centro de investigación y Docencia Económicas. (SEP/CIDE). (2010a). ENAVI. Encuesta Nacional de Vinculación en Instituciones de Educación Superior. Secretaría de Educación Pública / Centro de Investigación y Docencia Económicas (CIDE)/ (2010b), Encuesta nacional de vinculación en empresas, ENAVES, México: SEP/CIDE.

Recuperado de:

http://laisumedu.org/showBib.php?idBiblio=10412\&cates=Ciencia $\% 2 \mathrm{C}+$ tecnolog $\% \mathrm{EDa}+\mathrm{e}+\mathrm{inno}$ vaci\%F3n\&idSubCat=167\&subcates $=3 .+$ Informes + de + ciencia $\% 2 C+$ tecnolog $\%$ EDa+e+innovaci $\% \mathrm{~F} 3 \mathrm{n} \& \mathrm{ssc}=30 \& \mathrm{~m}=$ mail $1 \& \mathrm{p}=$ mail 1

Super, J. C. (1993). Los Orígenes de la Extensión Social en la Universidad Latinoamericana. Revista Universidades de la Unión de Universidades de América Latina, 6.

Torres, A., Dutrénit, G., Barrera, N., y Sampedro, J.L. (2009). Patrones de vinculación Academia- Industria: Factores determinantes en el caso de México. Sinnco. Recuperado de: https://www. researchgate.net/publication/228474318_Patrones_de_vinculacion_AcademiaIndustria_Factores_determinantes_en_el_caso_de_México

Universidad de Guadalajara/PROFMEX. (2014). Encuesta sobre vinculación de investigadores en ciencia y tecnología en México. México: UdG/PROFMEX.

Universidad Nacional de la Plata (2015). Extensión Universitaria, un compromiso con la integración. Recuperado de:

http://www.unlp.edu.ar/articulo/2008/4/10/extension_apertura_institucional Universidad 